\title{
AI BASED DIGITAL TRANSFORMATION FOR RACK STORAGE MANAGEMENT IN SMART FACTORY
}

\section{MILAN KUMAR, V. M. SHENBAGARAMAN \& N. PARTHIBHAN}

SRM Institute of Science and Technology, Kattankulathur, Tamilnadu, India

\begin{abstract}
Digital transformation leverages generation to make charge for several stakeholders, innovate and accumulate the abilities to rapidly adapt to changing situations. In any manufacturing storage or warehouse is one of the key activity as it stores the inventory. To make the full utilization of the warehouse space the racks are created, the artificial intelligence base rack storage management systems not only saves energy but also the time while doing the milk run for replenishment of material in the shop floor

Keywords: Digital Transformation, Artificial Intelligence, Smart Factory, Storage Management, machine learning, advance analytics
\end{abstract}

Received: Jun 08, 2020; Accepted: Jun 28, 2020; Published: Sep 24, 2020; Paper Id.: IJMPERDJUN20201396

\section{INTRODUCTION}

\section{- Digital Transformation}

Digital transformation is not nearly disruption or era. it's far about value, human beings, optimization and the functionality to rapidly adapt even as such is wanted thru a wise use of era and facts.

Digital transformation leverages generation to make charge for several stakeholders, innovate and accumulate the abilities to rapidly adapt to changing situations.

Digital transformation is predominantly performed in a enterprise context, it furthermore affects unique companies like governments, public area organizations and groups which might be worried in tackling societal demanding situations which consist of pollutants and growing older populations through leveraging one or greater of those gift and growing technology.

\section{- Artifical Intelligence \& Machine Learning}

Artificial Intelligence will not just be about automation or predictions. The wide variety of particular use times will increase at lightning speed and complicated algorithms are going to be required to execute those obligations. self sustaining automobile will become a mainstream truth. Self-programming strategies have already grow to be effective sufficient to form AI expand its non-public code; imparting massive opportunities to programmers for constructing not possible structures. The expanded optimization and pace of development in artificial Intelligence has induced its adoption in various industries at breakneck speed, along side hello-tech, telecom, car, economic provider and healthcare, to name some. This yr AI is anticipated to automate extra complicated techniques, offering ahead-looking intelligence and creating industrial organization value.

As cellular becomes even greater powerful with 5G, there will be ripple consequences in the course of the producing organization[1-4]. There may be advanced connectivity for robotics and automation inside the production 
facility and faster actual-time analytics from field operations.

\section{- Smart Factory}

The smart manufacturing unit is described as a production facility wherein physical production strategies and operations are mixed with virtual era, smart computing and large records to create a extra opportunistic device for agencies that target manufacturing and deliver chain control[5-6]. The smart factory unit are an thing of organization 4.zero, a modern day phase in the commercial enterprise Revolution that focuses closely on real-time information, embedded sensors, connectivity, automation, and machine studying.

As factories evolve in mild of the facts revolution, organizations want to reconsider how they manipulate everything from automation techniques to workforce improvement methods[7-9]. Alongside the manner, producers will want modernized gear, along with robust, bendy company aid planning systems as a data and transactional backbone that help them adapt quickly as they construct towards a smart-factory destiny.

\section{- Introduction of System}

The objective is to analyze and diagnose the anomalies in the system. A generic approach has been depicted in image below.

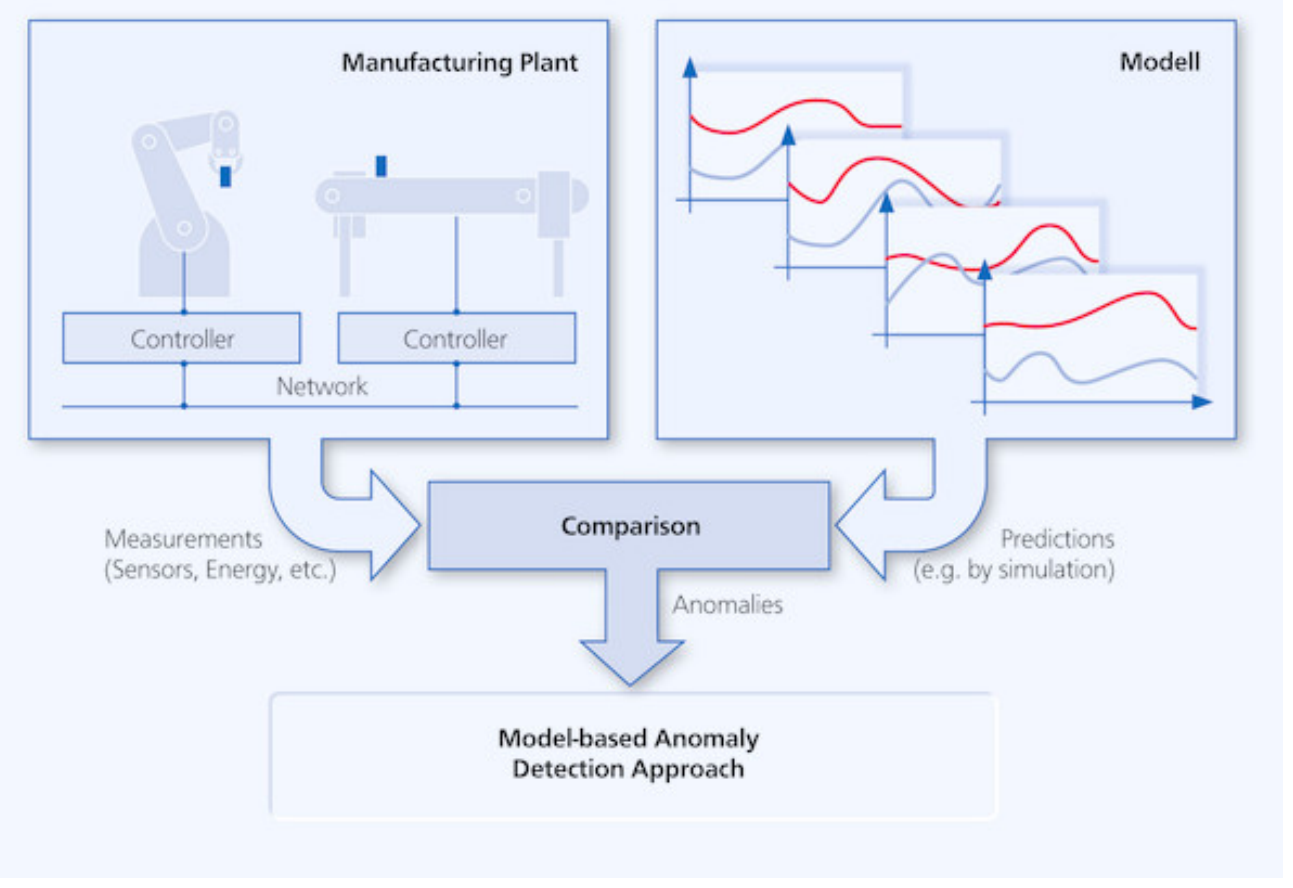

Figure 1

\section{Model based Analysis}

In this project, the HRS demonstrator transports wares (or objects) between its different shelves. There are two iterations to it - one is Standard iteration as a milk run and other is Optimized iteration to simulate energy optimization.

\section{Intelligent Energy Management for the Smart Factory (Industry 4.0)}

There are two aspects to understand the phenomenon of HRS demonstrator. One is Physical understanding of flow and second is data understanding of flow or how the data has been collected and measured? 


\section{- Physical-Flow Understanding}

The rail system demonstrator has 4 conveyors BRU, BHR, BHL, BLO and 2 rails HR, HL. The conveyors BRU and BLU can only move horizontally and whereas conveyors BHR and BHL can move both horizontally and vertically; with the help of rails. The quoted dimensions of all 4 conveyors are $640 \mathrm{~mm}$ x $85 \mathrm{~mm}$ x $297 \mathrm{~mm}$. Dimensions of rails HR and HL are not given. Each conveyor is driven by its own electric motor (power transmission to conveyors is by belts).

\section{- Data Extraction Technique}

The objective of this project (HRS demonstrator) is self-diagnosis and anomaly detection[9,10]. The technique used was to compare normal learned data with anomalous data (for both standard and optimized versions) to extract underlying data behavior models, anomalies, timings etc.

To achieve this objective each conveyor belt was fitted with three induction sensors. First sensor is at $36 \mathrm{~mm}$ from left edge, second at $266 \mathrm{~mm}$ from left edge and third at $36 \mathrm{~mm}$ from right edge. NO sensors had been mentioned for rails. So we may assume the sensors on conveyors BHR and BHL may have been used to measure data about the rails HR and HL.

Four data files of this projectare provided: Normal Standard, Normal Optimized, Anomalous Standard, and Anomalous Optimized.

\section{- Data}

Lets look into the data sets and various files. As first step I have uploaded all the files and various packages to help create the analyses.

\section{- HRS Data Glimpse}

In all 4 data sets there are same number and kind of variables. There are main 3 variables (or recorded data) are for each of the conveyors $(B R U, B H R, B H L, B L O)$ and rails $(H R, H L)$. Besides these variables are also accompanied with Time stamp and Labels.

The variables are as follows:

- Weg: Distance

- $\quad$ Power: Power

- Voltage: Volts

- Timestamp: Time elapsed in seconds to complete one forward-backward motion i.e. start from initial position 'i' at ' $\mathrm{t}=0$ ', on conveyor BRU and go to conveyor BLO and come back at initial position ' $\mathrm{i}$ '.

- $\quad$ Labels: ' 1 ' if there is an anomaly, or ' 0 ' otherwise.

\section{Sample Data}

\# Observations: 25,907\# Variables: 20\#Timestamp <dbl> 0.00000000, 0.04599762, 0.15100098, 0.20600128, ...\#Labels <int > $0,0,0,0,0,0,0,0,0,0,0,0,0,0,0,0, \ldots$ \#I_W_BLO_Weg<int> -107, -107, -107, -107, -107, -107, -107, -107, ...\#O_w_BLO_power $<$ int $>0,0,0,0,0,0,0,0,0,0,0,0,0,0,0,0, \ldots \#$ _.w_BLO_voltage $<$ int $>0,0,0,0,0,0,0,0,0,0$, $0,0,0,0,0,0, \ldots \#$ I_w_BHL_Weg $<$ int $>0,0,0,0,0,0,0,0,0,0,0,0,0,0,0,0, \ldots \#$ O_w_BHL_power $<$ int $>0,0,0,0,0$, 
$0,0,0,0,0,0,0,0,0,0,0, \ldots \# \mathrm{O} \_\mathrm{w} \_B H L \_v o l t a g e<$ int $>0,0,0,0,0,0,0,0,0,0,0,0,0,0,0,0, \ldots \# I \_$w_BHR_Weg<int $>$ -1268, -1268, -1268, -1268, -1268, -1268, -1268,... \#O_w_BHR_power $<$ int> 0, 0, 0, 0, 0, 0, 0, 0, 0, 0, 0, 0, 0, 0, 0, 0, ...\#O_w_BHR_voltage $<$ int $>0,0,0,0,0,0,0,0,0,0,0,0,0,0,0,0, \ldots$ I I_w_BRU_Weg<int > -26, -26, -26, 29, 29, 29, $107,107,107,107,1 \ldots \#$ O_w_BRU_power<int> 84, 84, 84, 21725, 21725, 21725, 23072, 23072, 2...\# O_w_BRU_voltage $<$ int $>11,11,11,54,54,54,56,56,56,56,56,56, \ldots$ \# I_w_HR_Weg<int $>0,0,0,0,0,0,0,0,0,0,0$, 0, 0, 0, 0, 0,...\# O_w_HR_power<int> 7168, 7168, 7168, 6726, 6726, 6726, 6726, 6726,...\# O_w_HR_voltage<int> 26,

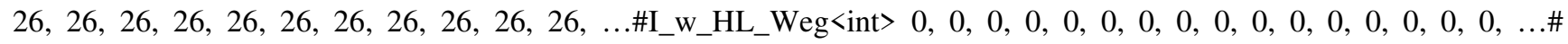
O_w_HL_power<int> 7720, 7720, 7720, 10756, 10756, 10756, 7408, 740..\#\# O_w_HL_voltage<int> 24, 24, 24, 26, 26, $26,24,24,24,24,24,24, \ldots$

\section{- HRS Data Cycles}

Each data-set has average +22000 rows (or observations). If you take timestamp as a reference point then we can calculate number of cycles per data set per simulation. Number of Cycles per data set is my first new feature.

\section{- HRS First Visual}

Visual of data showing Weg (or distance) on time stamp for all cycles. The Weg (or distance) has both positive and negative values.

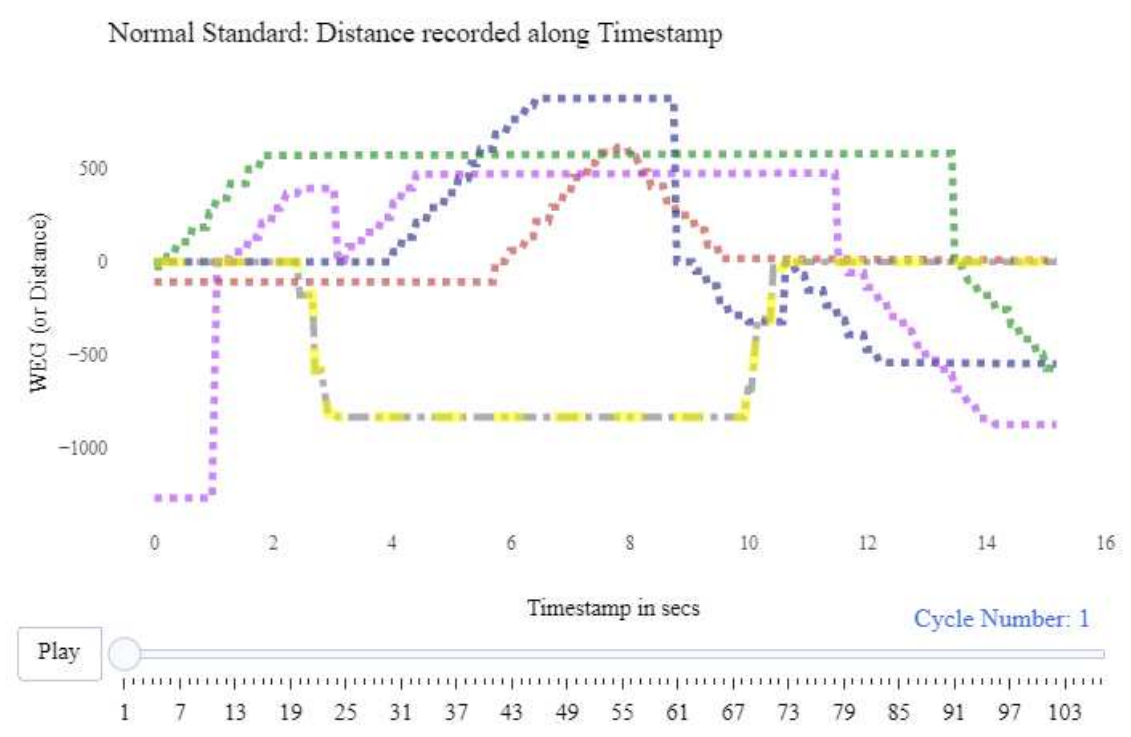

Figure 2

\section{- Variables}

There are 3 main variables Weg (or Distance), Power, and Voltage that had been recorded for all the conveyors and rails in addition to Timestamp and Labels.

Please Note: Sensors recorded the data. They showed the values of Weg, Power, Voltage, Timestamp at that time $=$ ' $\mathrm{t}$ ' and position $=$ 'i'.

Besides I have created more features for all the conveyors, rails and all the data sets - to visualize operations behavior as well.

- Total Power= 2 Power of Conveyors $+\sum$ Power of Rails 
- $\quad$ Time Gap=Timestamp-Timestamp-1

- $\Delta \mathrm{Weg}=\mathrm{Weg}-\mathrm{Weg}-1$

- $\Delta$ Power=Power-Power-1

- $\Delta$ Voltage $=$ Voltage - Voltage -1

- $\Delta$ Velocity $=$ Weg/Time Gap

- Weg (or Distance)

Apparently it seems it's the distance covered by a conveyor belts or rails to move the object and that was measured by sensors. But, if you'll observe in "Visuals: Weg vs Timestamp" graphs[11,12]. It's observed the measured data can be both positive or negative. Plus maximum values measured in Weg for any conveyor can even be greater than the length of individual each conveyor i.e. $640 \mathrm{~mm}$.

Normal Standard and Normal Optimized data's $\Delta$ Weg vs Time Gap variables were juxtaposed, as is in graph below, conveyor BHL data has shown big variations.

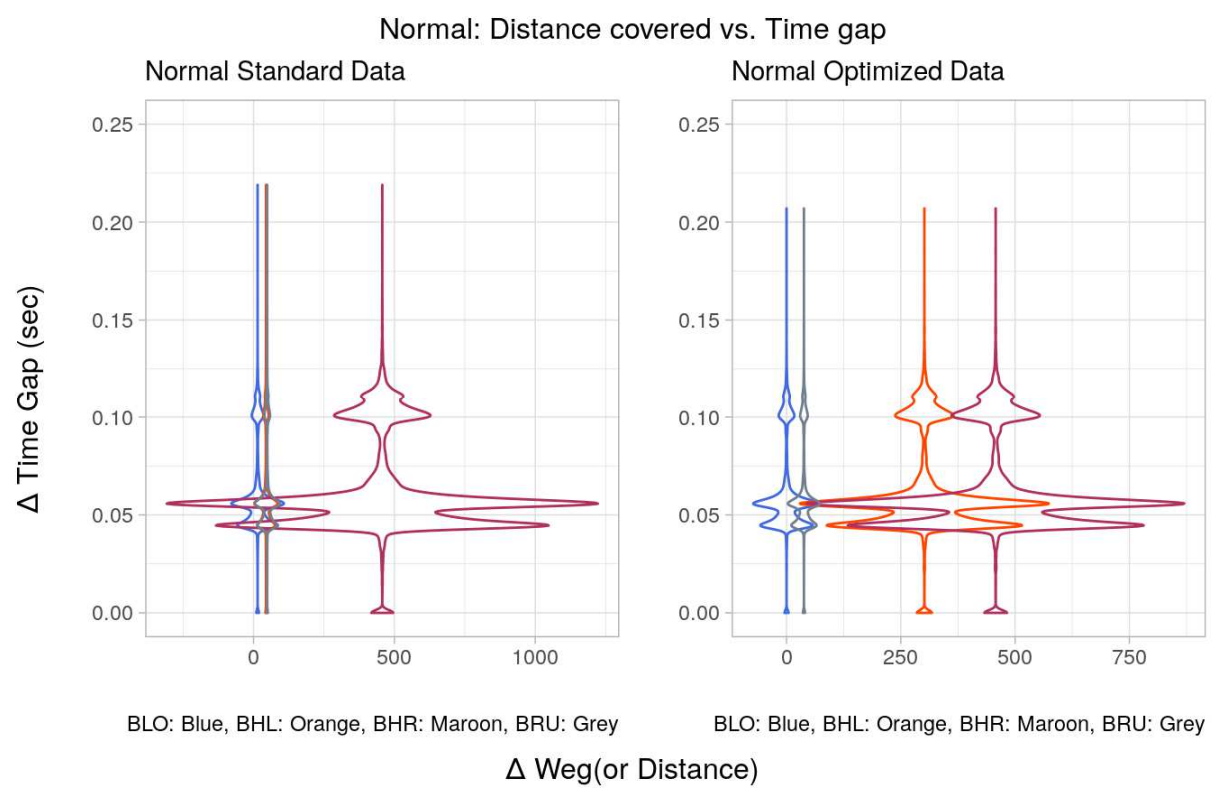

Figure 3

\section{- Power}

The variable Power plays a pivotal role in these data sets. Its relation with time to calculate Energy and later to optimize it is the objective behind it. Therefore, Sum of all the quoted values in all the Power variables gives the Power's behavior and total optimized power. 


\section{Normal Standard: Total Power distribution}

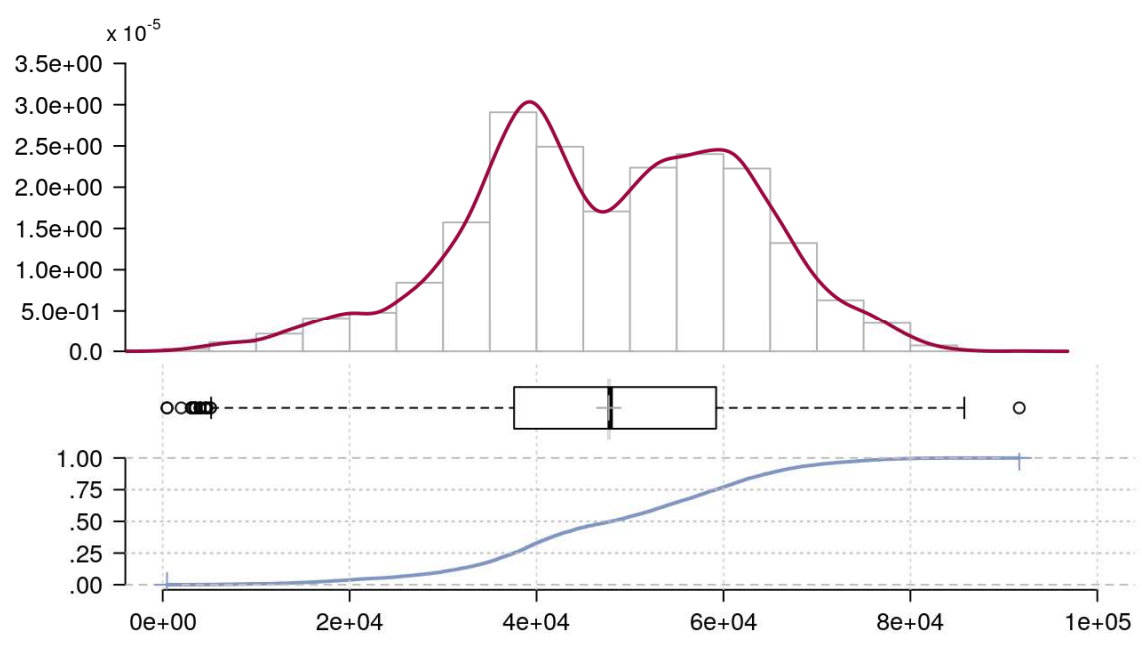

Figure 4

\section{- $\quad$ Power Variations - Both Negative \& Positive}

Power variations or $\Delta$ Power variables were created to see how the power changes with each recorded timestamp by the sensors? Does it shows similar pattern as that of physical movement of the object on conveyor or it has some other pattern?

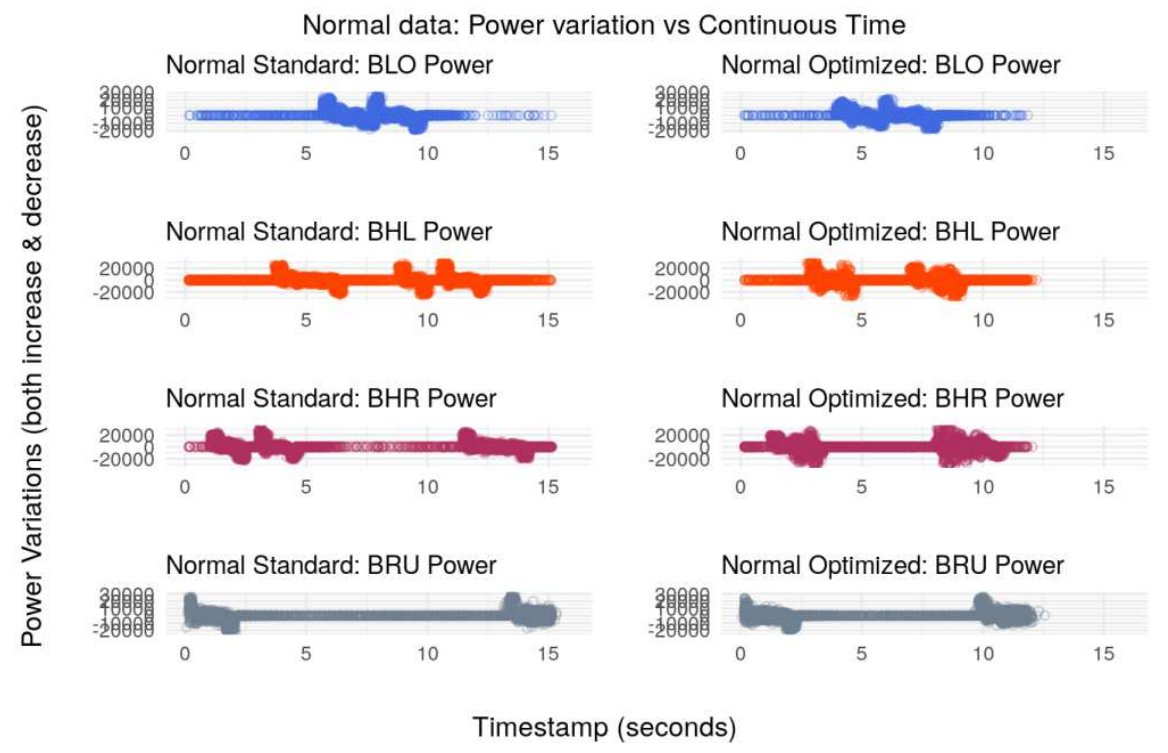

Figure 5

Normal Standard and Normal Optimized data's $\Delta$ Power vs Timestamp variables were juxtaposed. As is in graph above, the utilization of power by any conveyor is in coherence with when that specific conveyor would run in one its given cycle.

\section{- Voltage}

Voltage and Power are directly related. Ohms Law of electrical circuits. Voltage variations are presented in contour form see it's behavior pattern. 
Normal Standard and Normal Optimized data's $\Delta$ Voltage vs Timestamp variables were juxtaposed. As is in graph below, the variations in voltage shows same behavior as that of power and physical movement of an object on conveyor - in it's given cycle.

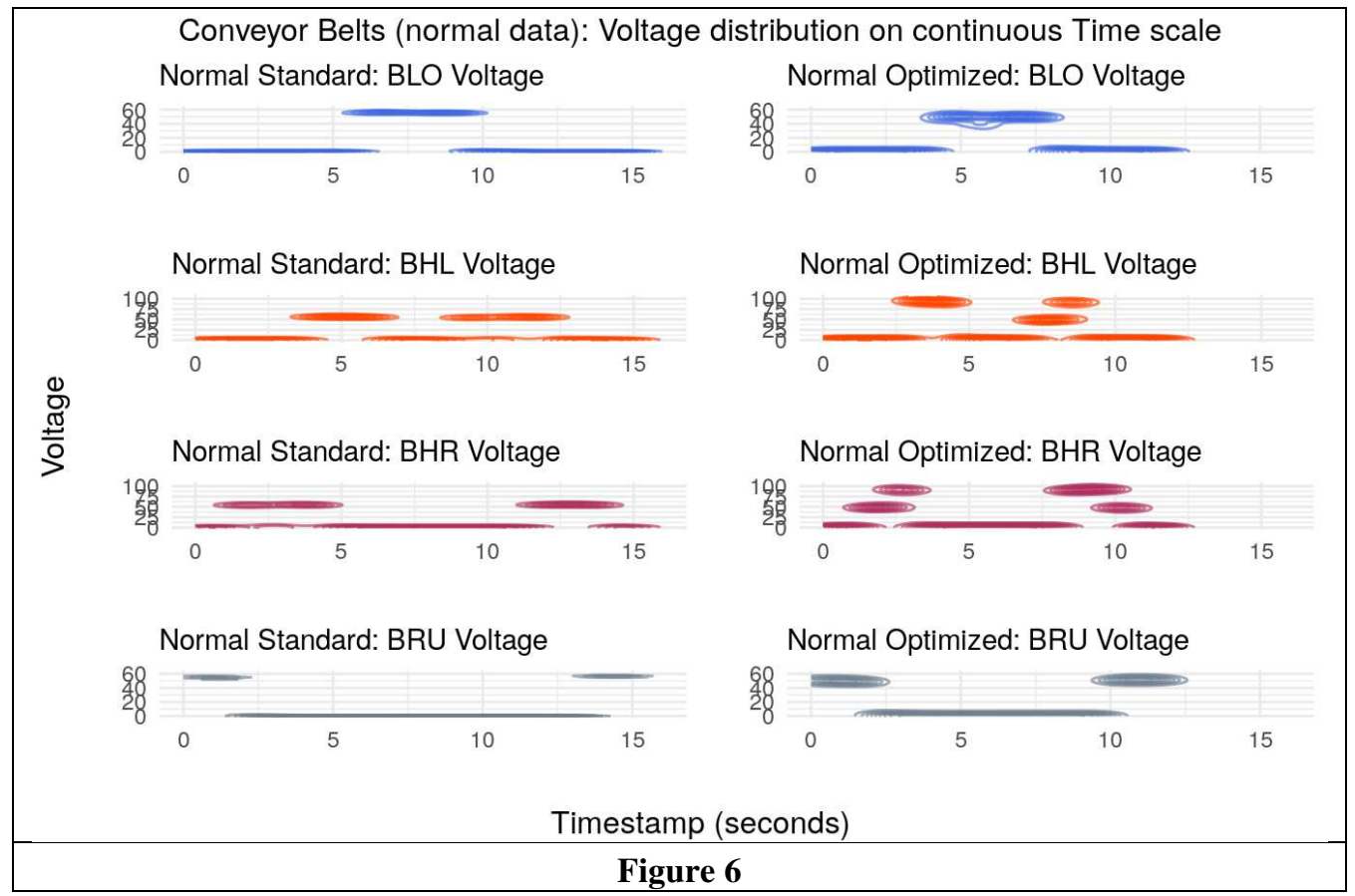

\section{- $\quad$ Timestamp and Labels}

Timestamp: Timestamp is in seconds. It starts at 0.0 seconds (recorded at up to eight decimal places) and ends anywhere between 15.0 seconds to 16.0 seconds. That said, each cycle has time duration of 15-16 seconds \&there are repetitions of cycles. These repetitions can be of 107 cycles if it's a Standard version data or 111 cycles if it's an Optimized version data set. OR we may say — as if it's a 15-16 seconds film caption repeated either 107 times or 111 times or more.

Labels: If categorical value is ' 1 ' then it is an anomaly else ' 0 '. The values of this variable distinguishes between Normal and Anomalous data sets.

\section{- Relationships}

In HRS demonstrator couple of conveyors are relying on rails for vertical movement of objects. This occurrence of physical coordination within sub-systems to optimize total energy may show some different behaviors and underlying models.

\section{- Data: Correlations}

In Normal Standard data's correlation:

- Voltage is positively and strongly correlated to Power for all the conveyors but not the rails.

- Distance variables of Conveyor and Rail combination are negatively correlated.

- Distance variable of both rails are positively and strongly correlated.

- Distance variable of all conveyors can be both positively or negatively correlated to their Powers. 
Ivui IIIai olaiıualu

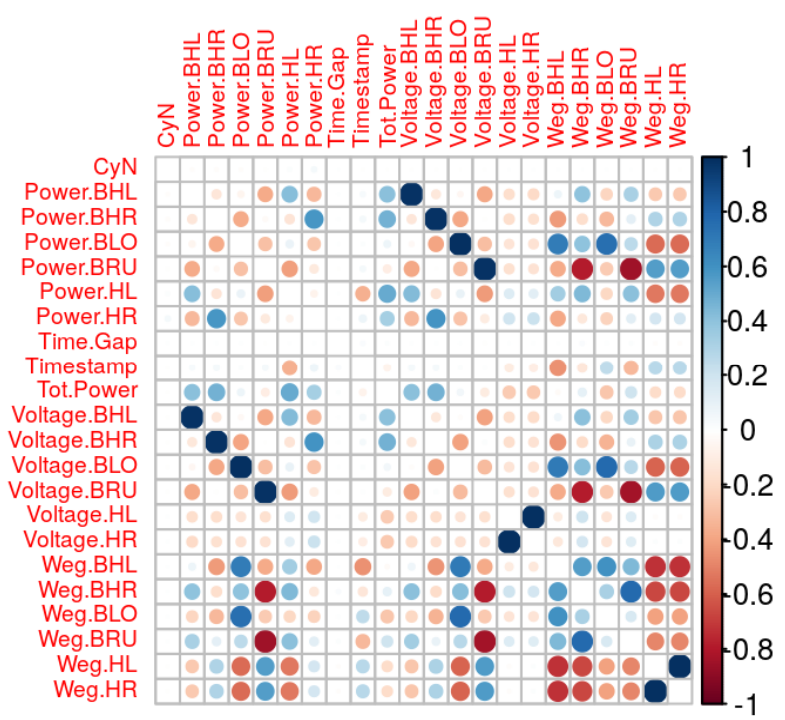

Ivul IIIal olailuaiu

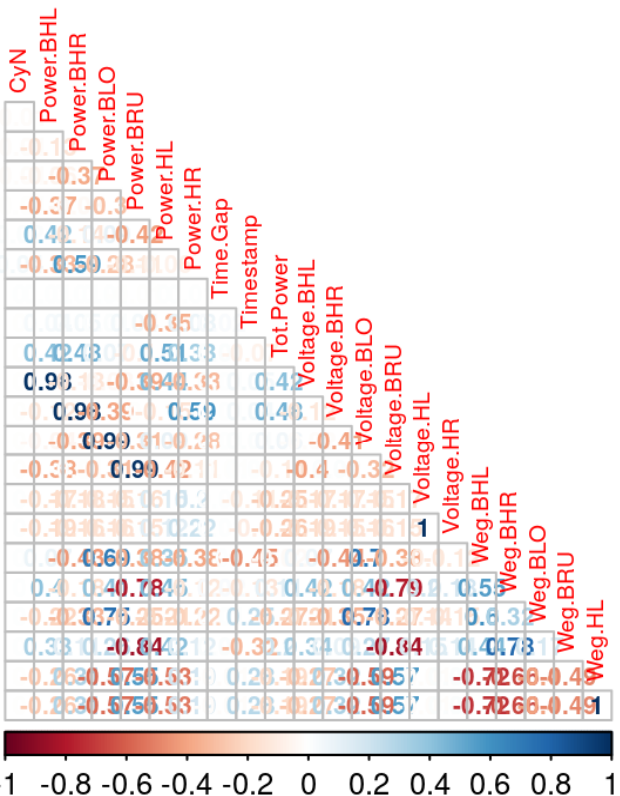

Figure 7

\section{- Physical Systems: Conveyor and Rails}

Conveyors BHL \& BHR are linked to rails HL \& HR. This combination or how they generate data can be seen in the graph below. $\Delta$ Powers of both the conveyors are taken less than ZERO[13]. This will look into the cases where Power of conveyors are increasing as it climbs the height with the help of rails.

Normal Standard: First rail HR does work to help climb the system and then rail HL does. Normal Optimized: First rail HL does work to help climb the system and then rail HR does.

But, Normal Optimized data for conveyor and rail combination is relative more jittery than the Normal Standard's data. 


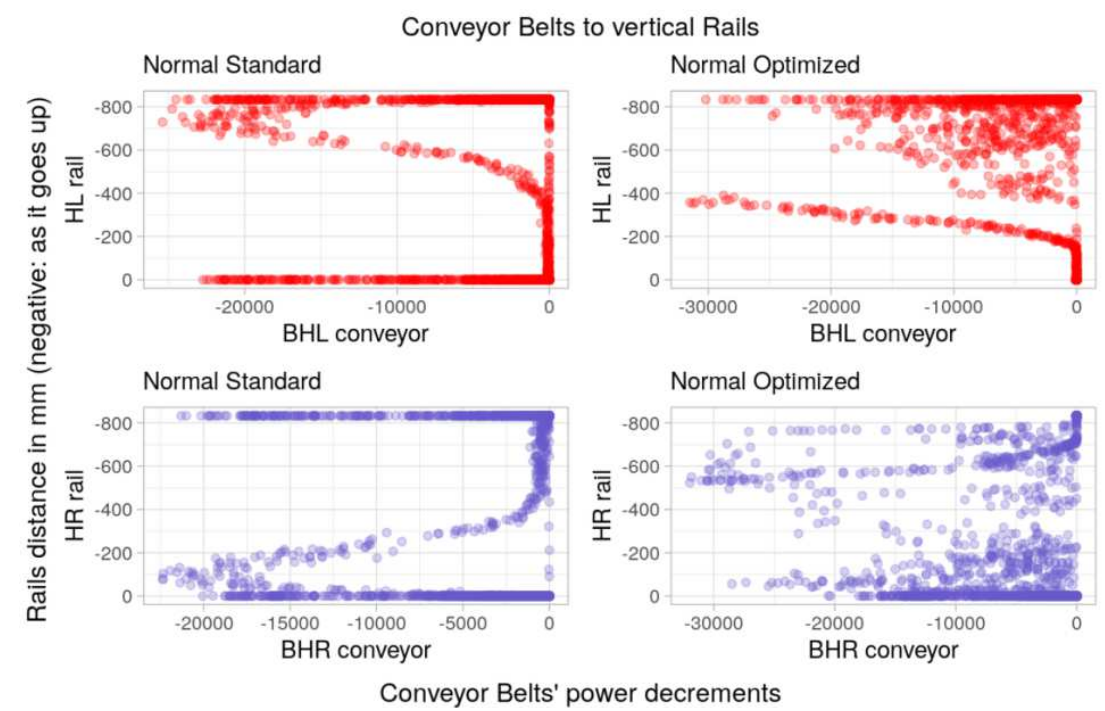

Figure 8

\section{- $\quad$ Cycles': Means + Standard Deviations}

In this section, Number of Cycles per data set takes precedence. We created new data sets[14]. Each data set has same original data set + Total Power as variables, but every value stated in these tables is it's mean per cycle and standard deviation per cycle.

\section{- Power}

Power plays again - this time at Cycle Number level. Let's see how the marker's dance?

\begin{tabular}{|l|l|l|l|l|}
\hline Conveyor or Rail & Mean(Max) & Mean(Min) & $\sigma($ Max $)$ & $\sigma($ Min) \\
\hline Power BLO & 5430 & 0 & 9754 & 8379 \\
\hline Power BHL & 7685 & 25 & 11154 & 9450 \\
\hline Power BHR & 6780 & 0 & 9676 & 8450 \\
\hline Power BRU & 8720 & 4403 & 10126 & 8733 \\
\hline Power HL & 14629 & 10756 & 8902 & 6981 \\
\hline Power HR & 12884 & 5972 & 8042 & 5519 \\
\hline
\end{tabular}

Figure 9 
POWER: Mean to Standard Deviation

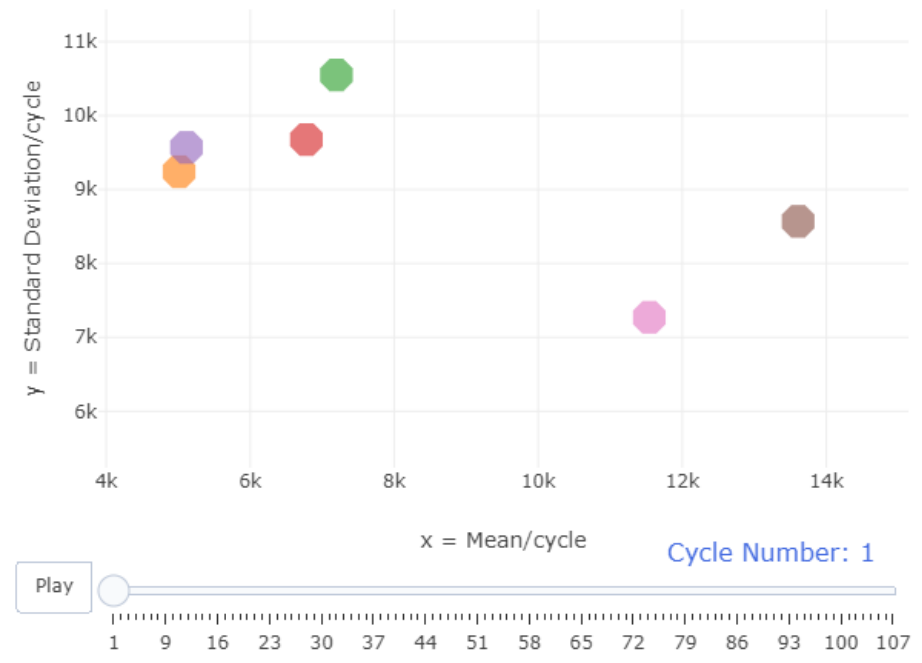

Figure 10

- Voltage

\begin{tabular}{|l|l|l|l|l|}
\hline Conveyor or Rail & Mean(Max) & Mean(Min) & $\sigma($ Max) & $\sigma($ Min) \\
\hline Voltage BLO & 13.66 & 0.00 & 23.23 & 21.92 \\
\hline Voltage BHL & 19.50 & 1.00 & 26.11 & 24.80 \\
\hline Voltage BHR & 18.84 & 0.00 & 25.67 & 24.43 \\
\hline Voltage BRU & 39.00 & 12.07 & 24.15 & 21.42 \\
\hline Voltage HL & 54.24 & 26.00 & 96.09 & 75.40 \\
\hline Voltage HR & 54.79 & 26.00 & 97.92 & 76.01 \\
\hline
\end{tabular}

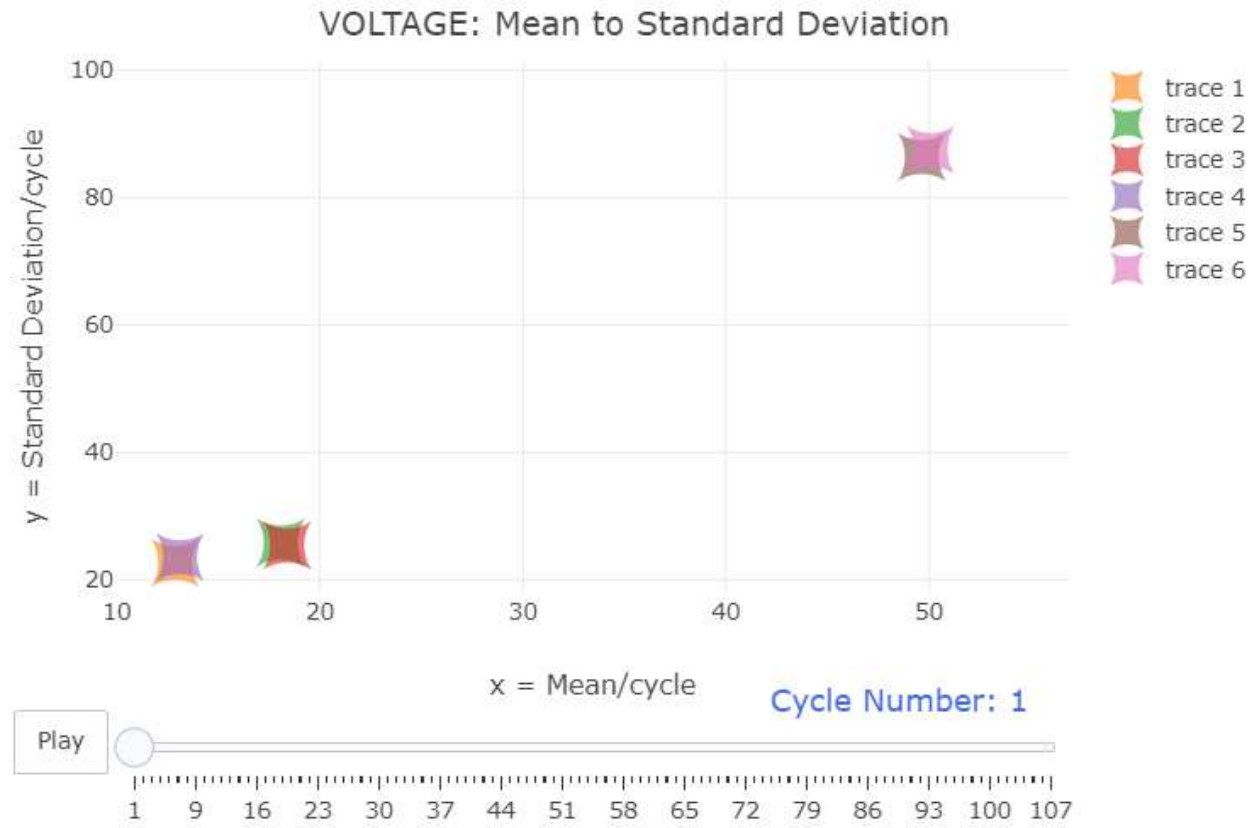

Figure 11 
- Power \& Weg (or Distance)

Zhang and Xia stated JIS B 8805 and Goodyear Tire \& Rubber Company's 1975 model are based on "Energy Conversion based model" methodology. Where Power of conveyor belts, under stationary conditions, can be divided into three parts.

- $\quad$ Power $_{\mathrm{ec}}$ : Power to run empty conveyor.

- Power $_{\mathrm{h}}$ : Power to move the material horizontally over certain distance.

- $\quad$ Power $_{1}$ : Power to lift the material a certain height. To this they add Power ${ }_{\text {acs }}$ as Power of accessories and defined Total Power as Power TT $=$ Power $_{\mathrm{ec}}+$ Power $_{\mathrm{h}}+$ Power $_{1}+$ Power $_{\mathrm{acs}}$ Lets see how Power of various conveyors and rails pan out with respect to Weg (or Distance) covered.

\begin{tabular}{|l|l|l|}
\hline Conveyor or Rail & Power(Median) & Weg (Median) \\
\hline BLO & 4939 & 43.99 \\
\hline BHL & 7030 & 42.23 \\
\hline BHR & 6187 & 42.33 \\
\hline BRU & 5101 & 429.70 \\
\hline HL & 13185 & -411.40 \\
\hline HR & 11292 & -411.50 \\
\hline
\end{tabular}

Figure 12

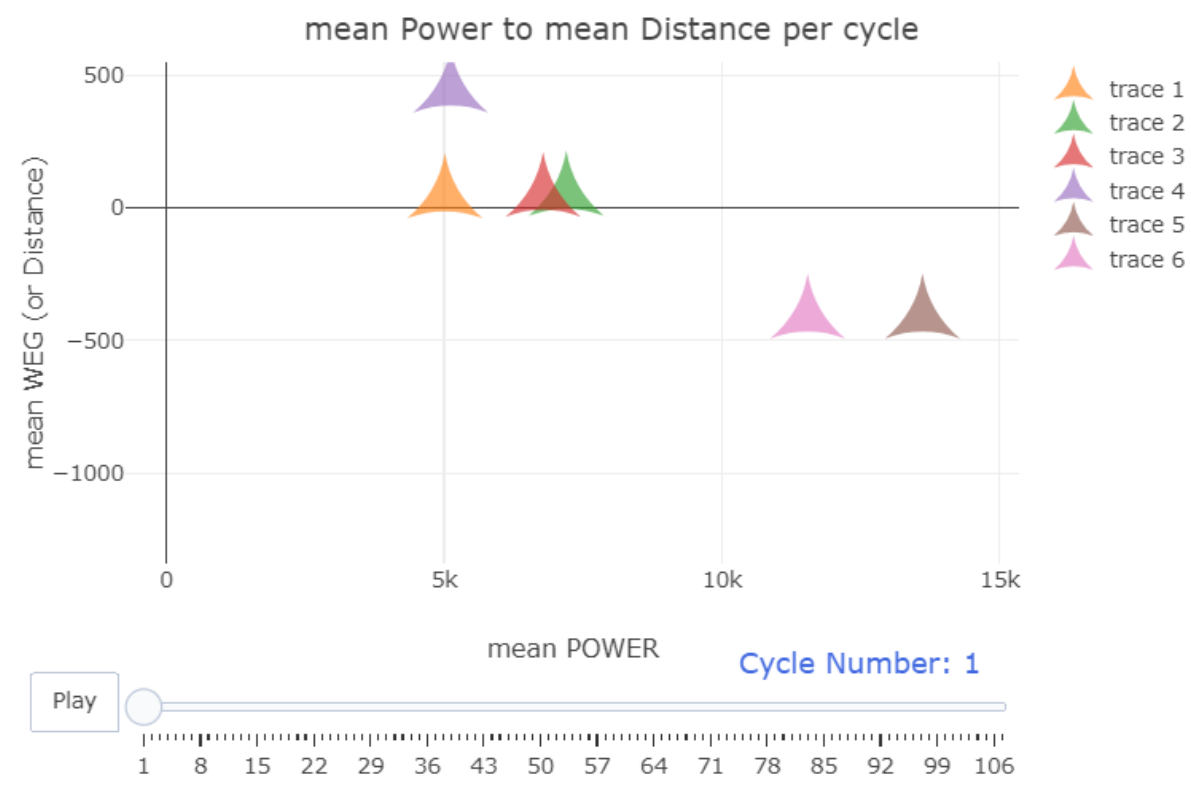

Figure 13

\section{- Tree-based: Total Power in Anomalies}

Anomalous Standard data set is more close to real life situation. So we have a task in hand that represent conditions and situations are is represented by the data. Now our objective is to draw efficiency for and in that cyber-physical system. Hence we do optimization to reach at - as per data — to a situation more like Anomalous Optimized data set.

In this section takes a deep dive into how the data quoted for Anomalies either actual or synthetic emerges. The approach I have taken is to Tree-based regression analysis. This is one of the easiest way to see the segmentation of data and understand the rationale behind it. In these sub-sections the objective is not to build an opitmization model or 
behaviour pattern, but only to demonstrate and to have better visual understanding of cyber-physical production systems. How step by step they are build?

\section{- Tree: Regression for Total Power}

In this Tree presentation, I show how Total Power of Anomalous Optimized data is linearly related to powers of each conveyor and rail.

\#box.palette "RdYlGn" (not diverging): \#FF7272 (near indianred1) to \#72FF89 (near seagreen1) \#cex 0.45 xlim c(0, 1) $\operatorname{ylim} \mathrm{c}(0,1)$

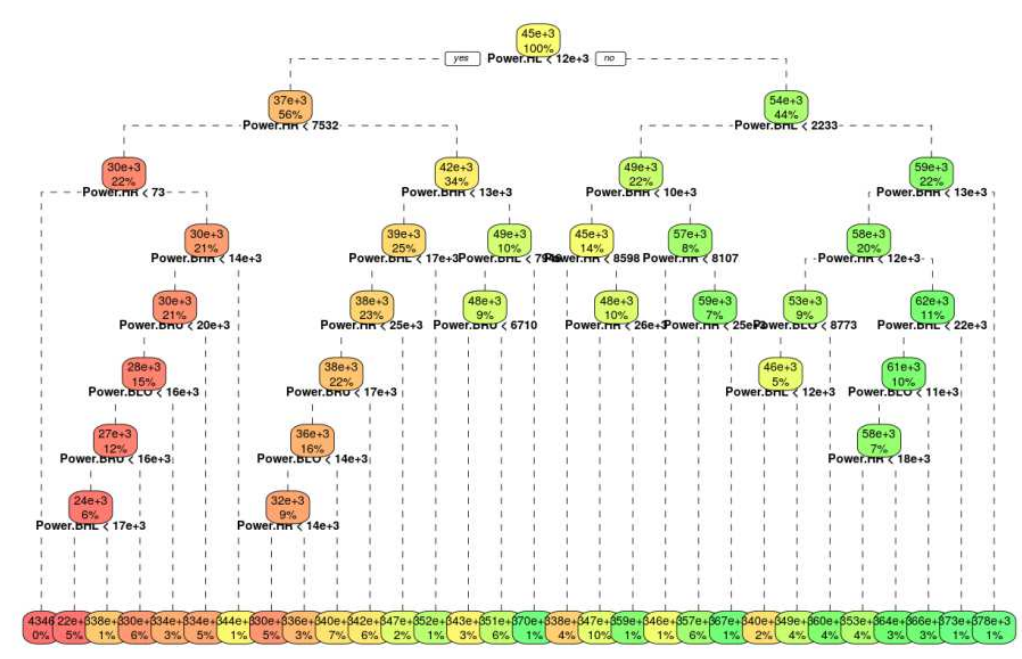

Figure 14

\section{- Tree Build-up}

Anomalies of Anomalous Optimized data has been taken. Total Power variable has been stated as objective to develop linear relation with all the power variables of Conveyors and Rails. As the Tree builds up, step by step, that section of tree turns navy blue in colour.

Please Note: This is only presentation and no conclusion has been drawn out of it. 

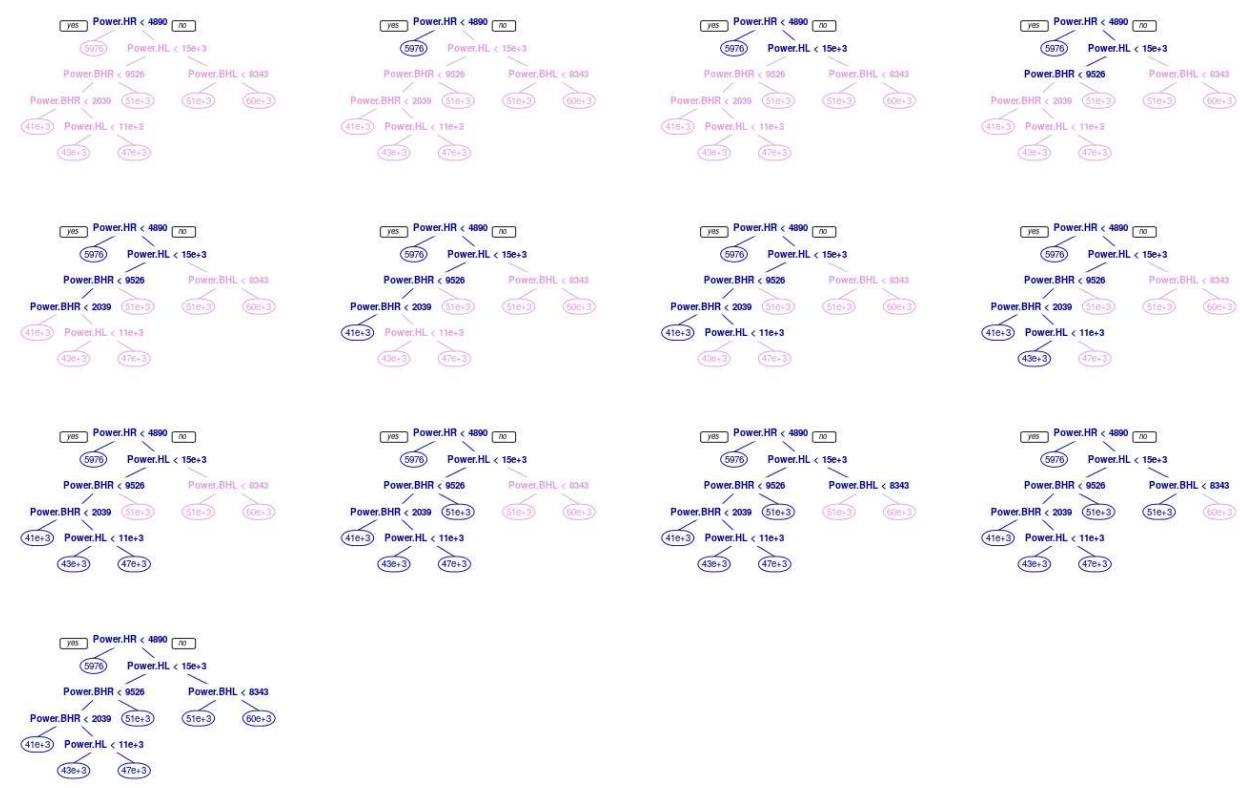

\section{- Tree: Graphical Representation}

Figure 15

In this graph Anomalies of Anomalous Standard data has been taken. The Total Power vs Timestamp variables has been shown. You'll observe the data with Anomalies is NOT spread across complete time horizon of 15-16 secs cycle. They have very short timestamp cycle.

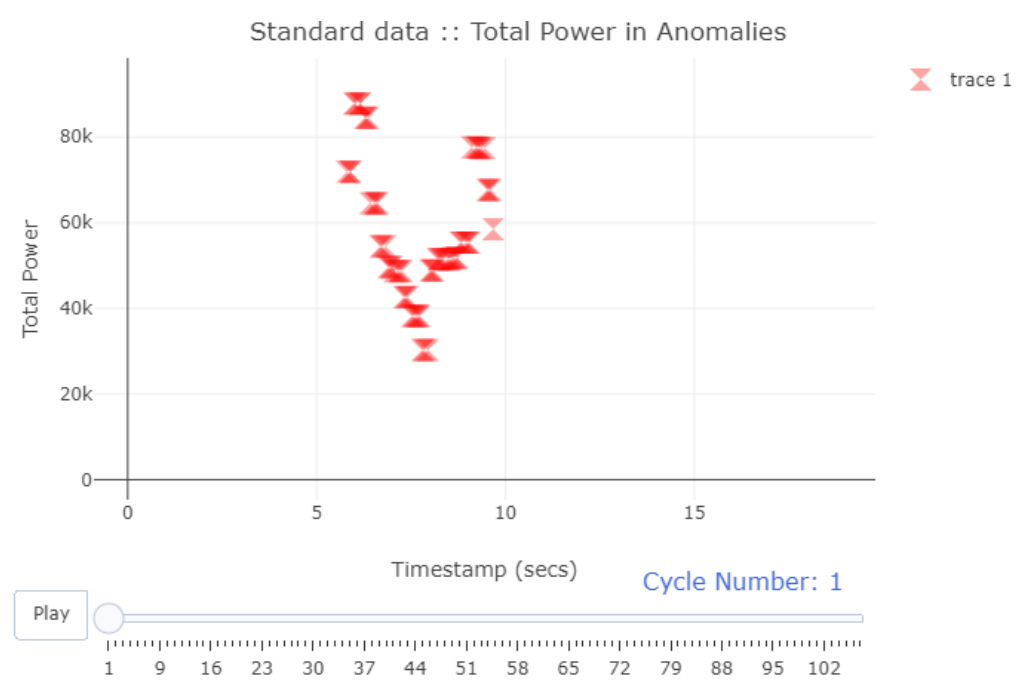

Figure 16

\section{- CONCLUSIONS}

This whole exercise emphasises on graphical presentation of the data of all 4 data sets. We tried pictorial presentation to explain CPPS' phenomenon, relationships between sub-systems, behaviour of data, patterns if any, and data generation etc.

In principle, the data recorded is in coherence with physical movement of object on conveyors and rails. A simple look at graphs of Power \& Voltage will show where the object was at a given timestamp. 
Each graph is replicated for all data sets to show variances of variables with respect to data generated form i.e. Normal vs Anomalous for Standard and Optimized versions and Standard vs Optimized for Normal and Anomalous versions.

This Artificial Intelligence (AI) based case can be applied for all kind of high rack storage scenarios leading to digital transformation in a smart factory manufacturing management.

\section{REFERENCES}

1. Lee YT, Kumaraguru S, Jain S, Hatim Q, Robinson S, Helu M, et al. A classification scheme for smart manufacturing systems'performance metrics. Smart Sustain Manuf Syst 2017;1(1):52-74.

2. Lalanda P, Morand D, Chollet S. Autonomic mediation middleware for smart manufacturing. IEEE Internet Comput2017; 21(1):32-9.

3. Lade P, Ghosh R, Srinivasan S. Manufacturing analytics and industrial internet of things. IEEE Intell Syst 2017;32(3):74-9.

4. Monostori L, Márkus A, Brussel HV, Westkämpfer E. Machine learning approaches to manufacturing. CIRP Ann ManufTechnol1996;45(2):675-712.

5. Wuest T, Weimer D, Irgens C, Klaus DT. Machine learning in manufacturing: advantages, challenges, and applications. Prod Manuf Res 2016;4(1):23-45.

6. Zhang W, Jia MP, Zhu L, Yan X. Comprehensive overview on computational intelligence techniques for machinery condition monitoring and fault diagnosis. Chin J Mech Eng 2017; 30(4):1-14.

7. R. N. Shaw, Pratima Walde and AnkushGhosh, "Enhancement of Power and Performance of 9x4 PV Arrays by a novel arrangement with shade dispersion", Test Engineering and Management, ISSN: 0193 - 4120 Page No. 13136 - 13146, 2020.9264 Milan Kumar, V. M. Shenbagaraman \& Ankush Ghosh Impact Factor (JCC): 8.8746 SCOPUS Indexed Journal NAAS Rating: 3.11

8. R. N. Shaw, PratimaWalde and AnkushGhosh, "Effects of Solar Irradiance on Load Sharing of Integrated Photovoltaic System with IEEE Standard Bus Network”, International Journal of Engineering and Advanced Technology, Volume 9 Issue-1, October 2019.

9. R. N. Shaw, PratimaWalde and AnkushGhosh, "A New Model to Enhance the Power and Performances of 4x4 PV Arrays with Puzzle Shade Dispersion”, International Journal of Innovative Technology and Exploring Engineering, Volume-8 Issue-12

10. Lu C, Wang Z, Qin W, Ma J. Fault diagnosis of rotary machinery components using a stacked denoising autoencoder-based health state identification. Signal Process 2017;130:377-88.

11. Chen Z, Deng S, Chen X, Li C, Sanchez RV, Qin H. Deep neural network-based rolling bearing fault diagnosis. Microelectron Reliab2017; 75:327-33.

12. Zhao R, Wang D, Yan R, Mao K, Shen F, Wang J. Machine health monitoring using local feature-based gated recurrent unit networks. IEEE TransaInd Electron 2018;65(2):1539-48.

13. Zhao R, Yan R, Wang J, Mao K. Learning to monitor machine health with convolution bi-directional LSTM networks. Sensors 2017; 17(273):1-18.

14. Wu Y, Yuan M, Dong S, Lin L, Liu Y. Remaining useful life estimation of engineered systems using vanilla LSTM neuralnetworks. Neurocomputing2017; 226(5):853-60. 